\title{
Nucleic acid cytokine responses in obese children and infants of obese mothers ${ }^{\text {is }}$
}

\author{
Kiva Brennan ${ }^{\mathrm{a}, \mathrm{b}}$, Bobby D. O'Leary ${ }^{\mathrm{c}}$, Danielle Mc Laughlin ${ }^{\mathrm{d}}$, David Kinlen ${ }^{\mathrm{a}, \mathrm{e}}$, \\ Eleanor J. Molloy, ${ }^{\mathrm{a}, \mathrm{b}}$, Declan Cody ${ }^{\mathrm{a}, \mathrm{e}}$, Sri Paran ${ }^{\mathrm{d}}$, Fionnuala M. McAuliffe ${ }^{\mathrm{c}}$, Andrew E. Hogan ${ }^{\mathrm{a}, \mathrm{g}, \mathrm{h}}$, \\ Sarah L. Doyle $\mathrm{a}^{\mathrm{a}, \mathrm{b}, *}$

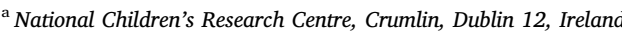 \\ ${ }^{\mathrm{b}}$ School of Medicine, Trinity College Dublin, Dublin 2, Ireland \\ ${ }^{\mathrm{c}}$ UCD Perinatal Research Centre, Obstetrics \& Gynaecology, School of Medicine, University College Dublin, National Maternity Hospital, Dublin, Ireland \\ d Department of Paediatric Surgery, Our Lady's Children's Hospital, Crumlin, Dublin 12, Ireland

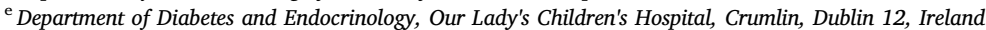 \\ ${ }^{\mathrm{f}}$ Coombe Women and Infants University Hospital, Dublin 8, Ireland \\ ${ }^{\mathrm{g}}$ Education and Research Centre and Conway Institute, St. Vincent's University Hospital, University College Dublin, Dublin 4, Ireland \\ ${ }^{\mathrm{h}}$ Institute of Immunology, Department of Biology, Maynooth University, Maynooth, Co Kildare, Ireland
}

\section{A R T I C L E I N F O}

\section{Keywords:}

Proinflammatory cytokine

Maternal obesity

Childhood obesity

Cytosolic nucleic acid

\begin{abstract}
A B S T R A C T
Almost a third of Irish children are now overweight and the country ranks 58th out of 200 countries for its proportion of overweight youths. With the rising obesity epidemic, and the impaired immune responses of this population, it is vital to understand the effects that obesity has on the immune system and to design future therapeutics, adjuvants and vaccines with overweight and obese populations in mind. Many current vaccines use adjuvants that have been found to be less effective at stimulating the immune response in children compared with adults and there is now substantial effort to design paediatric-focused adjuvants. Additionally, vaccine responses have been shown to be less effective in obese populations indicating that this is a particularly vulnerable population. We have recently identified cytosolic nucleic acids (CNAs), as novel candidate adjuvants for childhood vaccines. Here we investigated whether immune responses to these candidate adjuvants were adversely affected in infants born to overweight or obese mothers, and in overweight and obese children. Type I Interferon (IFN) and proinflammatory cytokines such as Tumor Necrosis Factor $\alpha$ (TNF $\alpha$ ) are vital for driving innate and adaptive immune responses. We found that childhood obesity conferred no significant adverse effect on CNA-induced Type I IFN responses when compared with lean children. Similarly, Type I IFN responses were intact in the cord blood of babies delivered from overweight and obese mothers, when compared with lean mothers. There was also no significant impact of obesity on CNA-induced TNF $\alpha$ responses in children or from cord blood of infants born to overweight/obese mothers. In all cases, there was a tendency towards decreased production of innate cytokine Type I Interferon and TNF $\alpha$, however there was no significant negative correlation. Interestingly, high maternal BMI showed weak and moderate positive correlation with IL-12p70 and IFN $\gamma$, respectively, in response to CNA stimulation. This study demonstrates that future adjuvants can be tailored for these populations through the use of activators of CNA sensors.
\end{abstract}

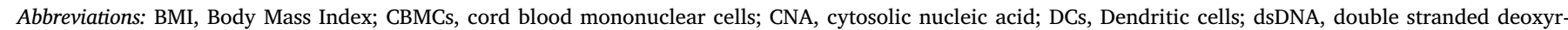

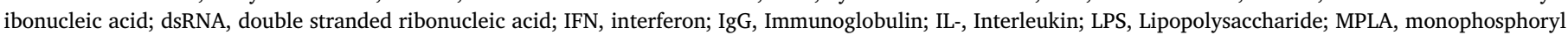

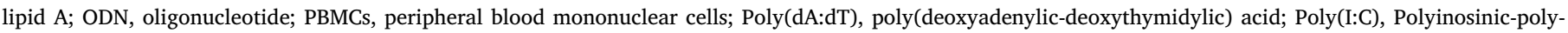

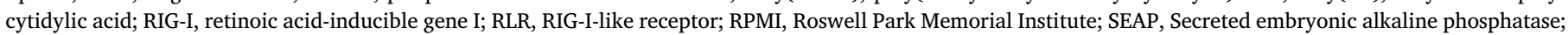
Th, T helper; TLR, Toll-like receptor; TNF, Tumor necrosis factor

This work was supported by The National Children's Research Centre (NCRC), Health Research Board HRA_PHR/2013/290 and Science Foundation Ireland 15/ CDA/3497.

* Corresponding author at: National Children's Research Centre, Crumlin, Dublin 12, Ireland and Department of Clinical Medicine, School of Medicine, Trinity College Dublin, Dublin 2, Ireland.

E-mail address: sarah.doyle@tcd.ie (S.L. Doyle). 


\section{Introduction}

Obesity has become a global epidemic over a period of only two decades. It now represents an urgent medical and a major societal challenge for which the solution certainly requires a multidisciplinary approach. Obese individuals are at higher risk of a wide range of infections including postoperative infections and other nosocomial infections, and also of developing serious complications resulting from common infections [1]. Obese individuals have been shown to be at increased risk of infection from certain vaccine-preventable diseases, such as tetanus, hepatitis B and influenza, due to poorer responses to vaccines in these individuals [2-4]. Although there is limited data concerning the effect of obesity on vaccine efficacy in neonates born to obese mothers, limited data from overweight and obese children suggest that responses to vaccinations are impaired in these groups $[2,5]$. Data from obese adults would also suggest that obesity increases the chances of a poor vaccine-induced immune response $[3,4,6,7]$. One study investigating the effect of increased body mass index (BMI) on vaccination responses showed that overweight children had reduced tetanus titers following vaccination when compared to lean children [2]. Another study in adults receiving Hepatitis B vaccination showed that obese adults have limited protection against the disease compared to lean adults [3]. Influenza vaccine responses are also affected by obesity, with increased risk of influenza among vaccinated adults who are obese [4]. Interestingly, initial antibody production is intact in obese individuals, however at later sampling, 12 months post vaccination, these antibody titers decreased below healthy weight individuals [7]. CD4 + and CD8 $+\mathrm{T}$ cell responses ex vivo were also decreased in

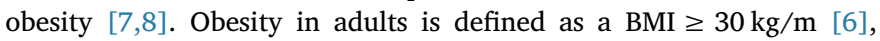
while for children it is more appropriate to use a BMI z-score. BMI zscores are measures of relative weight adjusted for child age and sex [5].

We have recently identified a potential novel adjuvant for childhood vaccines. In adults, Poly(I:C) or Poly(dA:dT), when transfected in to cells, activate cytosolic nucleic acid (CNA) sensors and induce Type I Interferon (IFN) production along with other proinflammatory cytokines such as Tumor Necrosis Factor $\alpha$ (TNF $\alpha$ ) [9]. It has previously been shown that while other activators of the innate immune system such as Lipopolysaccharide (LPS) and CpG, TLR4 and 9 ligands respectively, function well in adults, they are less able to activate the immune system of neonates and young children [10-13]. Adding to this defective paediatric immune response to TLRs, maternal obesity has been shown to lead to diminished responses to TLR ligands in cord blood monocytes and dendritic cells, when compared to cord blood of lean mothers [14], suggesting that adjuvants targeting these pathways [15-17] would be less efficient in neonates born to obese mothers. Cytosolic Poly(I:C) and Poly(dA:dT), unlike many Toll Like Receptor (TLR) agonists, can activate the neonatal immune response as efficiently as in adults, thereby opening up the possibility of harnessing these responses for broader use in adjuvant development.

In this study, we wished to investigate whether the CNA responses previously observed in neonates and children were intact in neonates born to overweight or obese mothers, and in overweight and obese children.

\section{Materials and methods}

\subsection{Subjects}

Umbilical cord blood samples were obtained from term births following normal pregnancy, labour and delivery at National Maternity Hospital (NMH), Holles St, Dublin 2. All infants had an uncomplicated postnatal course and Apgar scores of 9 at $5 \mathrm{~min}$. Maternal BMI at booking appointment was recorded. A BMI of 18.5-24.9 was classified as healthy, BMI between 25 and 29.9 was classified as overweight, while a BMI of $>30$ was classified as obese. Venous blood from infants, children and adolescents of various ages was collected during elective surgical procedures, where no indication of infection or underlying genetic or endocrine disease was present, (such as hydrocele repair, umbilical hernia repair, onychocryptosis, orchiopexy repair) in Our Lady's Children's Hospital Crumlin (OLCHC), Dublin 12. In all cases children had an extra 1-10 $\mathrm{ml}$ of blood drawn post-anaesthetic, thus avoiding extra venupuncture. Children's BMI, age and sex at blood draw was recorded and BMI z-scores were calculated. Volumes drawn were age and weight dependent, according to standard, approved guidelines. Ethics approval was obtained from the Ethics Committees of $\mathrm{NMH}$ and OLCHC and informed consent was obtained from each subject or their parent/guardian. Adult blood samples were from healthy adults, supplied by the Irish Blood Transfusion Service.

\subsection{Isolation of mononuclear cells}

Primary PBMCs or CBMCs were isolated from healthy human blood or cord blood. Cells were cultured at $37^{\circ} \mathrm{C}, 5 \% \mathrm{CO} 2,95 \%$ air in RPMI1640 , with stable $2.5 \mathrm{mM}$ L-glutamine and $0.5 \mathrm{mM}$ sodium pyruvate with 10\% FBS (all from Sigma-Aldrich).

\subsection{Stimulation of PBMCs and CBMCs}

Poly(I:C) $(5 \mu \mathrm{g} / \mathrm{ml})$ (Invivogen) and Poly(dA:dT) $(5 \mu \mathrm{g} / \mathrm{ml})$ (SigmaAldrich) were transfected into PBMCs or CBMCs using TransIT-X2 (Mirus).

\subsection{Measurement of cytokines}

HEK Blue TNF $\alpha / \mathrm{IFN} \alpha / \beta$ Assays were performed as per manufacturer's instructions using Quanti-Blue Detection Reagent. SEAP levels were determined using a spectrophotometer plate reader at $630 \mathrm{~nm}$. BioLegend LEGENDplex ${ }^{\mathrm{TM}}$ Human Inflammation Panel (13plex) was carried out as per manufacturer's instructions to determine levels of IFN $\gamma$ and IL-12p70. A BD LSR Fortessa cell analyser was used to acquire samples and BioLegend LEGENDplex ${ }^{\mathrm{TM}}$ software was used for analysis.

\subsection{Statistical analyses}

Data was analysed with GraphPad Prism software. Normality testing was carried out using Shapiro-Wilk and D'Agostino \& Pearson omnibus normality testing. When datasets were found to follow a non-normal distribution a Kruskal-Wallis with Dunn's multiple comparison test or Mann Whitney $U$ test was carried out for comparison of groups. For correlation analysis, Pearson correlation coefficient was used when datasets were found to follow a Gaussian distribution, while datasets with non-normal distribution were tested for correlation using Spearman's rank correlation. Two-tailed tests were used throughout, and the statistical approaches were all deemed to be valid for each individual experiment.

\section{Results}

It is widely accepted that obesity has immune complications, both co-morbid disease and inflammation, and immune dysregulation, and can often lead to decreased immune response to infection or vaccination [18]. It is also well established that the innate immune systems of children do not always function in the same manner or with the same efficiency as adults $[19,20]$. Deficiencies in the responses to TLR activation in children is an example of how the immune system of children and adults differs $[12,13]$. We have recently reported that Poly(I:C) and Poly(dA:dT), activators of the cytosolic nucleic acid sensors of the innate immune system, can activate the immune systems of neonates and infants as efficiently as in adults [9]. Given that many of the adjuvants in current use rely on activating TLR pathways, we hypothesise that 
Table 1

Characteristics of maternal lean, overweight \& obese study group.

\begin{tabular}{|c|c|c|c|}
\hline Characteristic & Lean & Overweight & Obese \\
\hline BMI, mean (SD), $\mathrm{kg} / \mathrm{m}^{2}$ & $22.35(1.82)$ & $27.5(1.38)^{\text {w.t.ke }}$ & $32.5(2.10)^{k, k \times k}$ \\
\hline $\mathrm{BMI}$ range & $17.84-24.96$ & $25.34-29.75$ & $30-36.64$ \\
\hline Maternal Age, mean (SD), years & $36.5(2.87)$ & $34.93(4.68)$ & $33.66(4.89)$ \\
\hline $\begin{array}{l}\text { Neonatal Birthweight, mean (SD), } \\
\text { kg }\end{array}$ & $3.49(0.57)$ & $3.87(0.55)$ & $3.76(0.57)$ \\
\hline Fetal Sex, M:F Ratio (n number) & $28: 21(47)$ & $14: 8(22)$ & $6: 8(14)$ \\
\hline
\end{tabular}

$* * * \mathrm{p}<0.005$, compared to Lean.

Poly(I:C) or Poly(dA:dT) may be candidates for future childhood vaccines. We were interested in investigating the effect of obesity on the Poly(I:C) and Poly(dA:dT) responses of neonates and children. We therefore re-analysed previously studied cord blood data in the context of BMI, and also added a cohort of overweight or obese children to previously acquired data on childhood responses to CNA. Characteristics of our study groups used to investigate the effect of maternal obesity on neonatal responses are presented in Table 1.

Mimicking viral infection through activation of cytosolic RLRs/ dsDNA sensors with transfected Poly(I:C) and Poly(dA:dT) resulted in equivalent Type I IFN production in CBMCs regardless of whether maternal BMI was in the healthy, overweight or obese range (Fig. 1A and $\mathrm{C}$ ). Correlation analysis confirmed our grouping analysis, showing

A

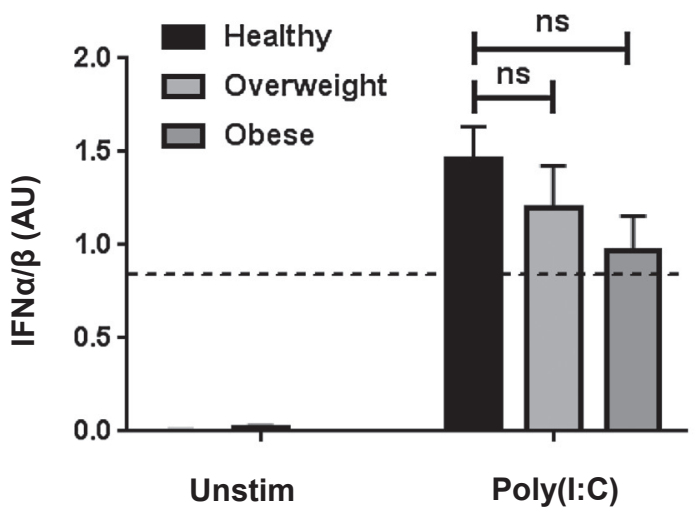

C

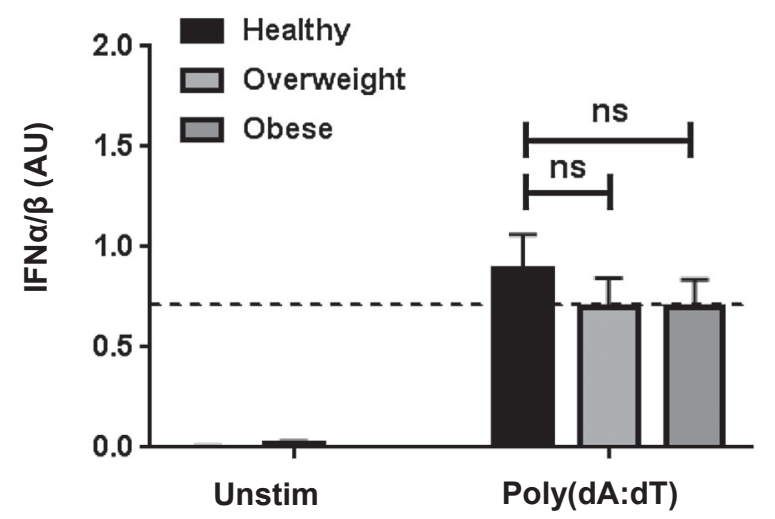

no positive or negative correlation between BMI and Poly(I:C)- or Poly (dA:dT)-induced Type I IFN responses (Fig. 1B and D). We had previously published that cytosolic Poly(I:C)-induced Type I IFN levels in CBMCs were higher than levels induced in adult PBMCs [9]. Here, stratification of responses by maternal BMI grouping demonstrates that this boost in immune stimulation is lost with increasing BMI, however levels of IFN production never drop below adult levels previously shown (0.842 AU \pm 0.092) [9], as illustrated by the dashed lines on Fig. 1A.

When TNFa was measured in response to Poly(I:C) or Poly(dA:dT) transfection, in CBMCs from babies delivered from mothers with different BMIs, we again observed no significant difference in cytokine production between CBMCs from healthy, overweight or obese mothers when grouped according to BMI (Fig. 2A and C). Similar to Type I IFN production, levels of TNF $\alpha$ production tend to decrease with increasing BMI groups, though not significantly and no negative correlation was found between maternal BMI and levels of CNA-induced TNF following correlation analysis. Levels of TNF $\alpha$ production in response to CNA also remain greater than or equal to previously observed adult levels (Poly (I:C): $33.65 \mathrm{pg} / \mathrm{ml} \pm 0.8 .58$, Poly(dA:dT): $48.51 \mathrm{pg} / \mathrm{ml} \pm 14.18$ (dashed lines Fig. 2A and C)) [9], regardless of maternal BMI. Taken together these results suggest that maternal BMI, while not having a negative correlation with innate cytokine output, does have the effect of blunting the cord blood responses to transfected Poly(I:C) or Poly (dA:dT) thereby eliminating the previously observed enhancement in
B

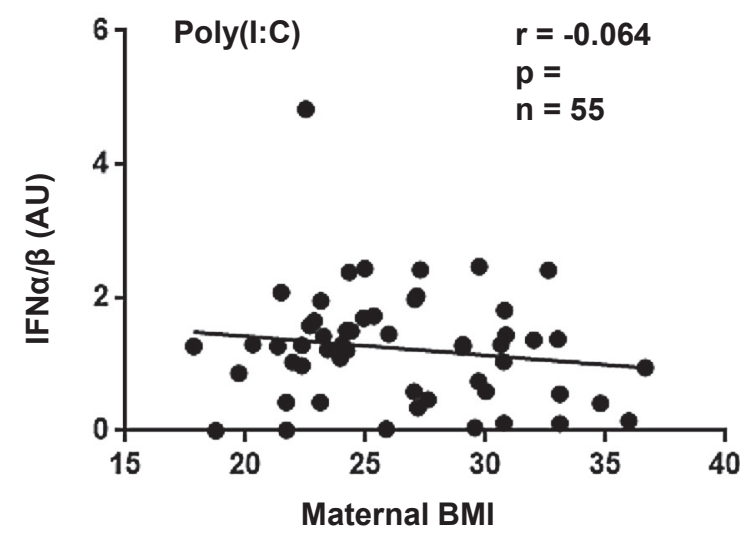

D

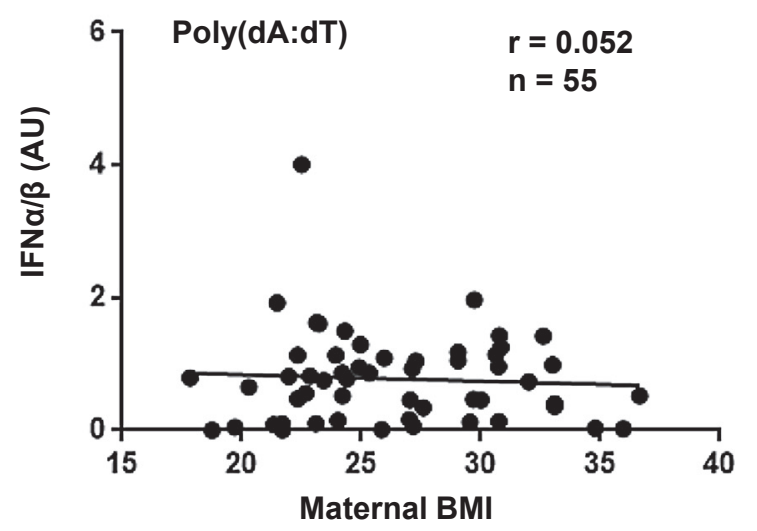

Fig. 1. Relationship of Type I IFN production in CBMCs to increasing maternal BMI. Neonatal cord blood mononuclear cells (CBMCs) from babies born to healthy, overweight or obese mothers were stimulated with (A and B) Poly(I:C) or (C and D) Poly(dA:dT) transfection for $24 \mathrm{~h}$. Levels of IFN $\alpha / \beta$ were assayed via HEK-Blue ${ }^{\mathrm{TM}}$ IFNa/ $\beta$ SEAP assay. (A and C) Data are mean \pm SEM ( $n \geq 14$ donors in each group). Kruskal-Wallis non-parametric test with Dunn's post-test was used to compare groups. (B and D) Correlation analysis of Type I IFN production and maternal BMI was carried out using Spearman's rank correlation testing. Dashed line represents adult Type I IFN production following relevant stimulation. 
A

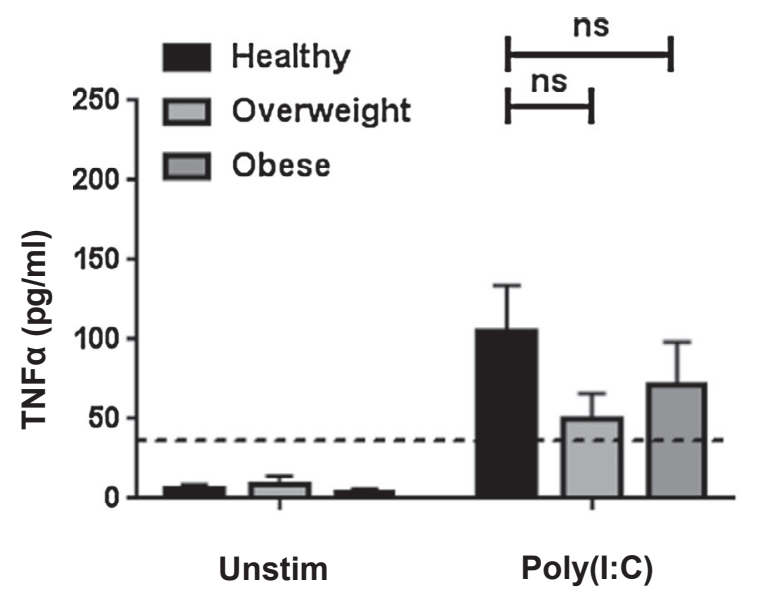

C

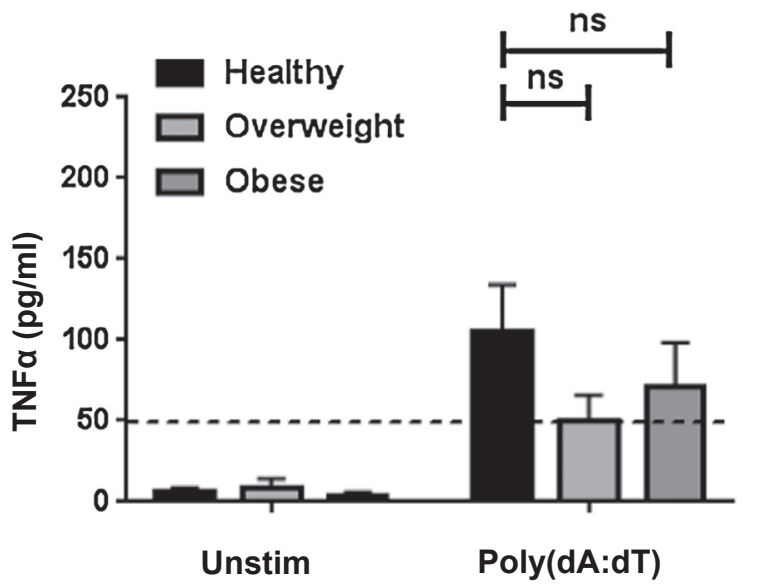

B

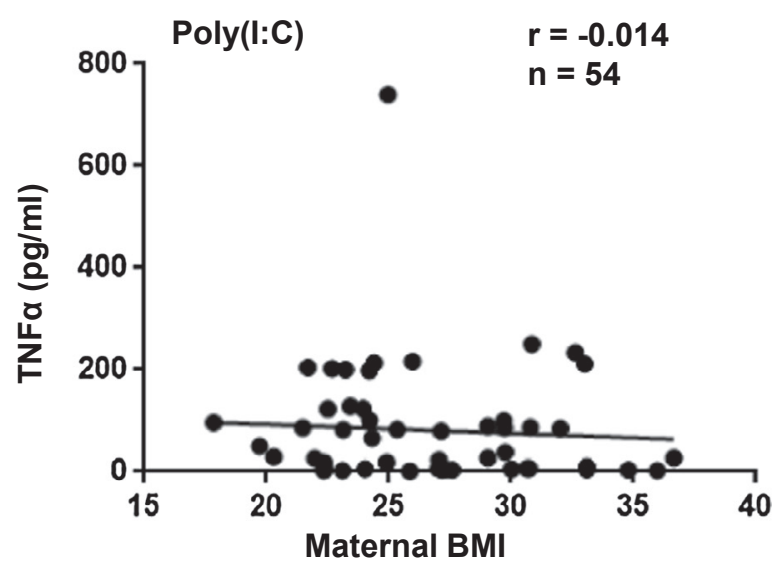

D

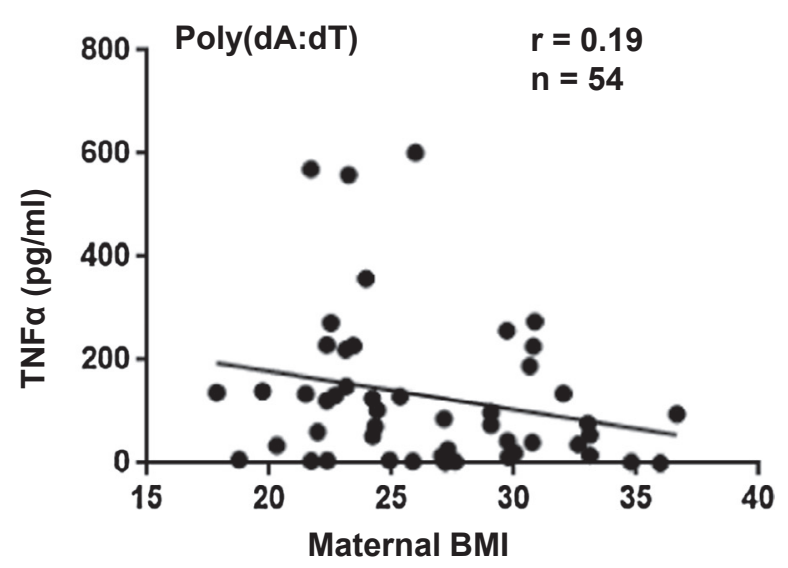

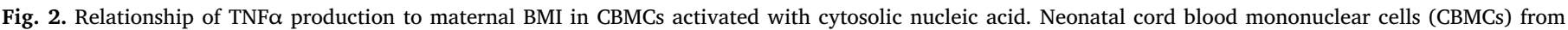

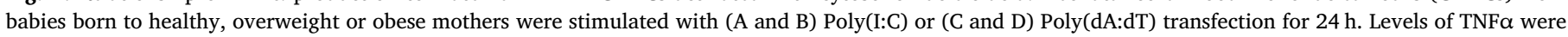

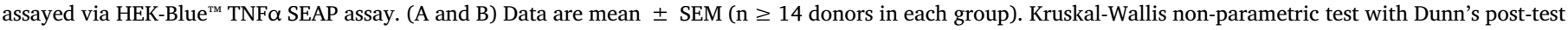

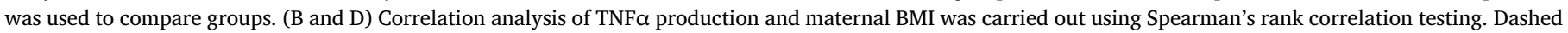
line represents adult TNF $\alpha$ production following relevant stimulation.

cytokine production in healthy CBMCs when compared with lean adult PBMCs [9]. Notably, maternal BMI does not affect the cord blood responses to transfected Poly(I:C) or Poly(dA:dT) to such a degree that it inhibits cytokine responses to below that of an adult.

We were also interested in whether obesity in childhood had an effect on Type I IFN and TNFa responses to transfected Poly(I:C) and Poly(dA:dT). Characteristics of our study groups used to investigate the effect of paediatric obesity on cytokine responses are presented in Table 2.

Interestingly, we found that while there was a slight tendency towards decreased Type I IFN production in overweight/obese children, there was no significant difference in Type I IFN production between

Table 2

Characteristics of lean \& overweight/obese paediatric groups.

\begin{tabular}{llll}
\hline Characteristic & Lean & Overweight/Obese & P value \\
\hline BMI z-score, mean (SD) & $0.06(0.67)$ & $2.29(0.52)$ & $<0.001$ \\
Age, mean (SD), years & $12.21(2.99)$ & $13.13(2.66)$ & 0.35 \\
Fetal Sex, M:F ratio & $12: 3$ & $10: 10$ & N/A \\
\hline
\end{tabular}

our healthy and overweight/obese groups in response to either Poly (I:C) or Poly(dA:dT) transfection (Fig. 3A and B). We also tested the TNF $\alpha$ responses of healthy or overweight/obese children to Poly(I:C) or Poly(dA:dT) transfection and found that there was no significant difference in the levels of TNF $\alpha$ produced regardless of BMI z-scores (Fig. 3C and D). In all cases, there was no correlation between BMI and CNA-induced cytokine production.

Type I IFN and TNF $\alpha$ can promote a Th1-type immune response which is highly desirable in shaping immunity in response to intracellular bacteria. Due to the potential of Poly(I:C) transfection to induce substantial Type I IFN and TNFa in neonates regardless of maternal BMI, we sought to explore whether Poly(I:C) could induce IL12p70, another important Th1-polarising cytokine, in the cord blood of babies delivered from overweight/obese mothers. Fig. 4A shows IL12 p70 production in CBMCs from overweight/obese mothers in response to Poly(I:C) compared to CBMCs from healthy weight mothers. There was no significant difference between the groups but when analysed for correlation, IL-12p70 production was found to positively correlate with BMI (Fig. 4B). IFN $\gamma$, considered both an innate and adaptive cytokine, is the primary cytokine that defines Th1 cells and is 
A
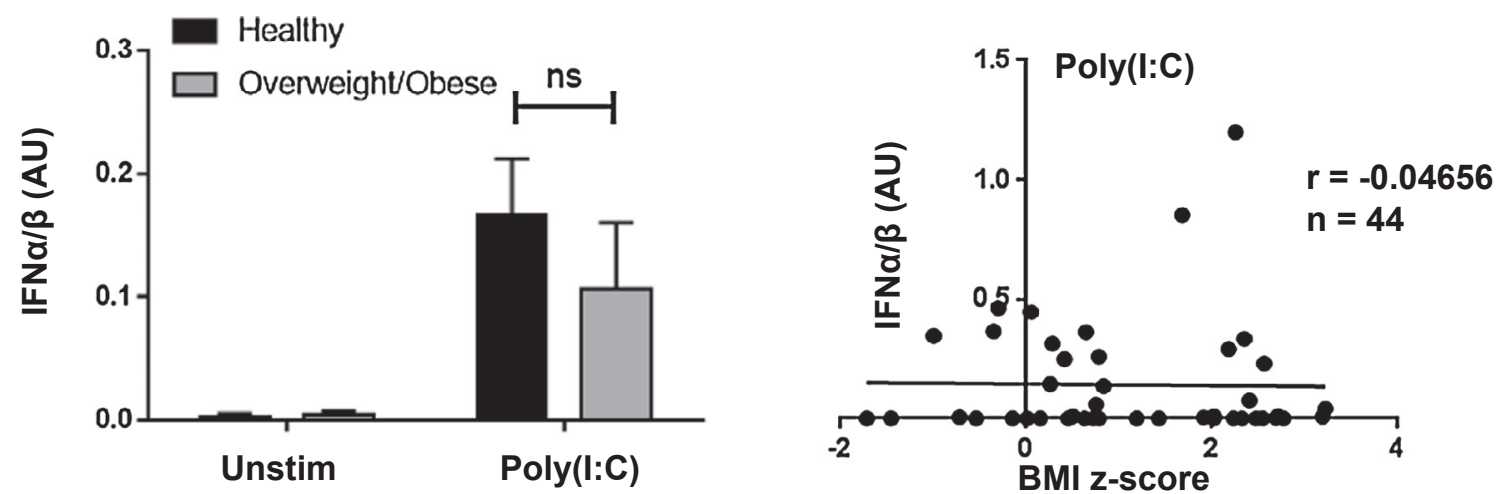

B
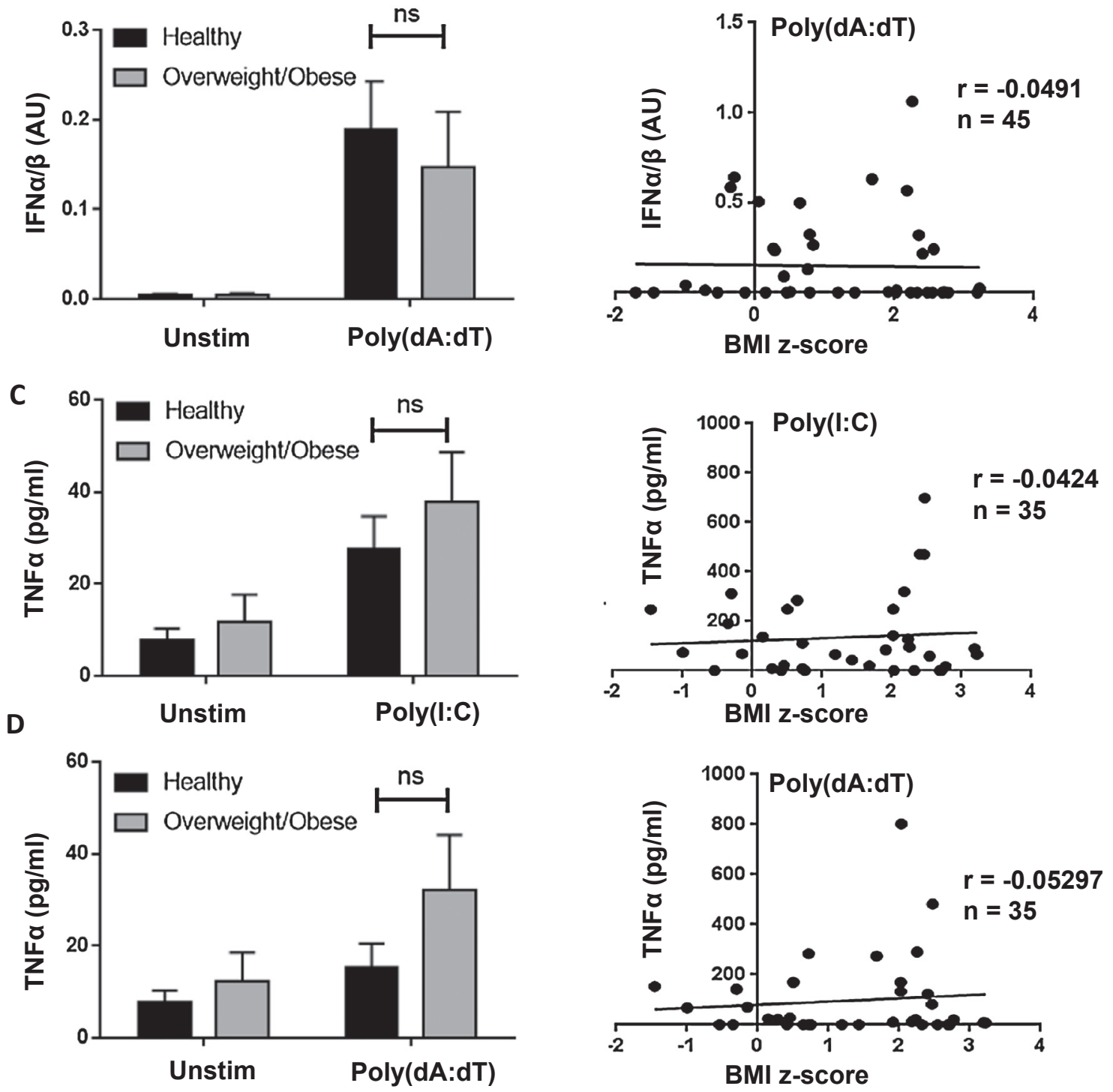

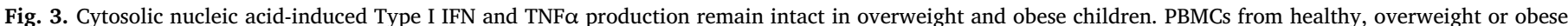

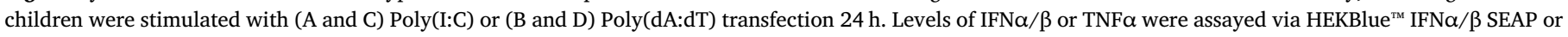

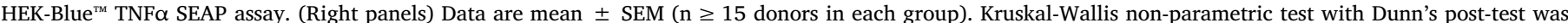

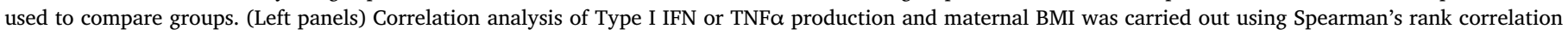
testing. 


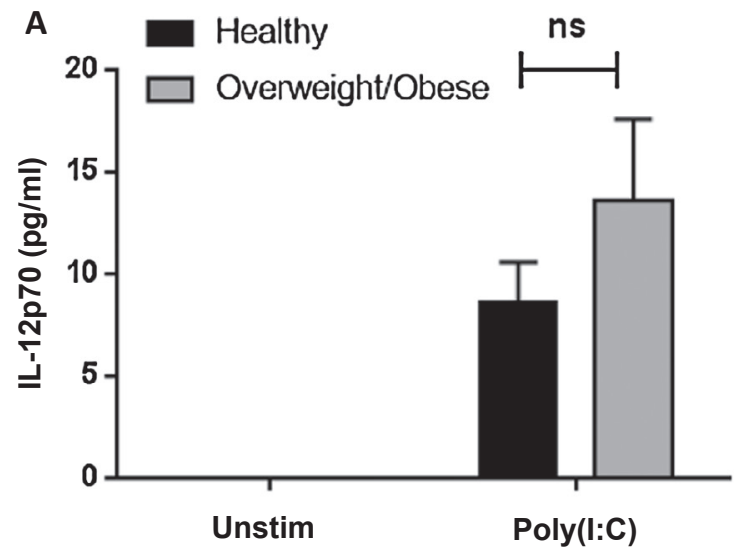

C

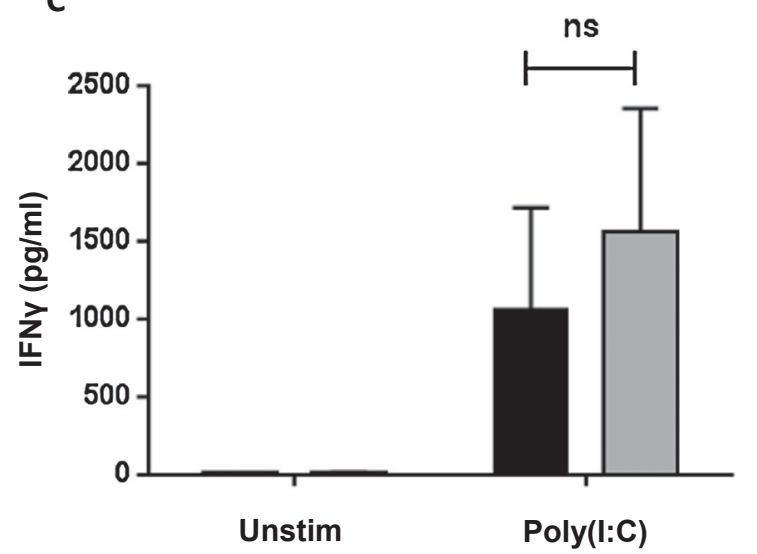

B

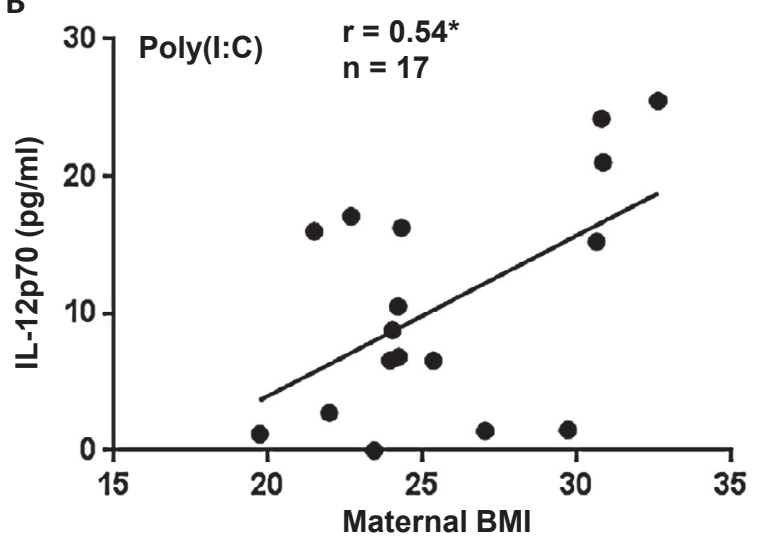

D

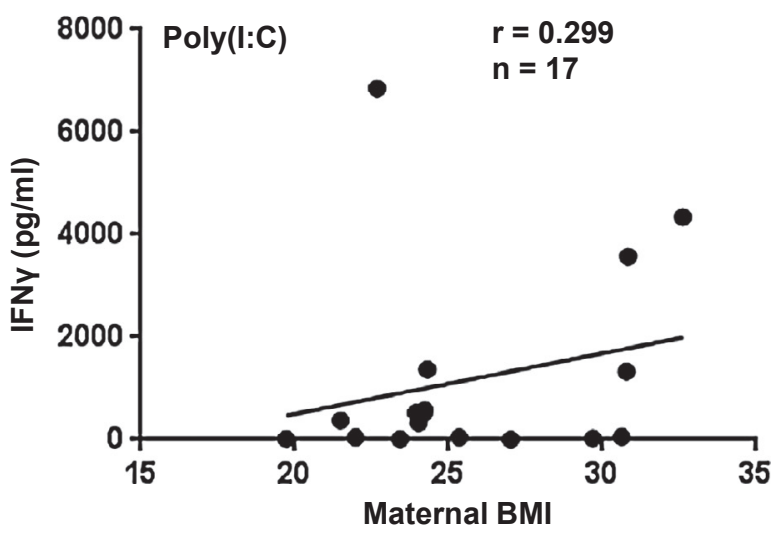

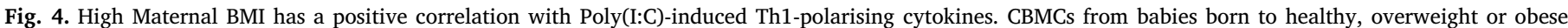

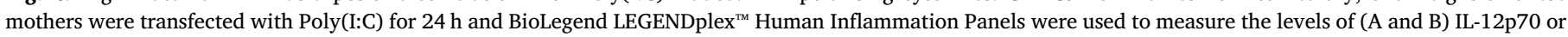

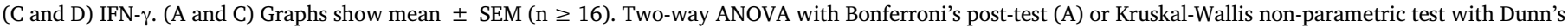

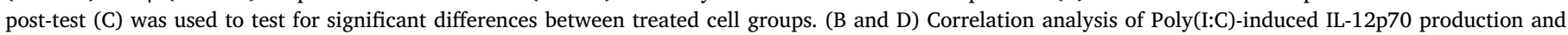

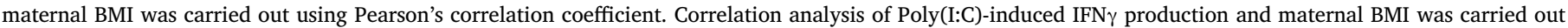
using Spearman's rank correlation testing. ${ }^{*} \mathrm{p}<0.05$.

predominantly produced by $\mathrm{CD} 4^{+} \mathrm{Th} 1$ and $\mathrm{CD} 8^{+}$cytotoxic $\mathrm{T}$ cells, driving further differentiation of $\mathrm{T}$ cells into Th1 cells. Interestingly, we found that IFN $\gamma$ production in the cord blood of babies, in response to Poly(I:C) transfection, was slightly increased when samples were grouped according to BMI (Fig. 4C). Further analysis showed that IFN $\gamma$ also correlates positively with BMI (Fig. 4D)

\section{Discussion}

Most literature evaluating the effect of obesity on vaccine efficacy has been carried out in adults. Obesity impacts negatively on vaccine efficacy and has been shown to give rise to an inflamed phenotype, including increased levels of circulating proinflammatory cytokines [18]. Most vaccines are given to children in the first months \& years of life, when obesity of the child has not become a factor in vaccine efficacy. However, studies have shown dysregulated immunity in obese children of school going age, an age when important booster vaccinations are given [21]. While trends in obesity continue to rise, women tend to be more prone to obesity than men $[22,23]$. As the frequency of obesity in young women increases, so too does the importance of our understanding the effects of obesity on the offspring during and postpregnancy.

Maternal obesity as a factor in vaccine efficacy has not been reported on to date. Given the fact that obesity is a risk factor for decreased vaccine efficacy in adults, it is possible that maternal obesity could affect early vaccine responses in their infants, as levels of circulating cytokines are similar in mothers and their offspring, early in life [24-28]. Importantly, maternal obesity has been linked to decreased cord blood monocyte and dendritic cell responses to TLR activation, including decreased TNF $\alpha$ production to LPS [14]. The importance of being able to efficiently produce proinflammatory cytokines, such as TNF $\alpha$ and Type I IFN, in response to infection or vaccination is well recognised. Type I IFNs activate cellular immunity by activating DCs and driving their maturation. This DC maturation allows antigen presentation to $\mathrm{CD}^{+} \mathrm{T}$ cells, cross-priming $\mathrm{CD} 8^{+} \mathrm{T}$ cells, and induction of IFN $\gamma$ and opsonizing antibodies [29,30]. Initiating Th1 responses and cytotoxic $\mathrm{T}$ cells is a major target in vaccine development. Having previously observed robust Th1 responses to cytosolic Poly(I:C) and Poly(dA:dT) in cord blood samples, we were interested in evaluating any impact of increased maternal BMI on these responses. Our initial measurements of Type I IFN and TNFa found no correlation of increasing maternal BMI on cytokine responses, or indeed increasing childhood BMI on PBMC responses. We tested the potential of cytosolic Poly(I:C) to promote this innate Th1 response and to encourage an adaptive response through measuring the production of Th1-polarizing IL-12p70 and induction of IFN- $\gamma$ in CBMCs from healthy and overweight/obese women. Interestingly, we found a moderate positive correlation of IL-12p70 production with maternal BMI suggesting that 
neonates from overweight/obese mothers might be better able to induce a cellular immune response to Poly(I:C). Similarly, IFN $\gamma$ production correlated positively, albeit weakly, with maternal BMI. While the effect of maternal obesity on vaccine responses in neonates has not yet been studied, a previous report has observed decreased CD4 T-cells in neonates born to obese mothers along with reduced monocyte and dendritic cell responses to TLR ligands [14]. The limited studies examining the effect of obesity on vaccination in children have focused on Hepatitis B and influenza vaccines and have shown deficiencies in the response to these vaccines in obese children. A recent paper from $\mathrm{Wu}, \mathrm{Y}$ et al. has shown that LPS-induced cytokine responses are decreased in PBMCs from obese children compared to lean children [31]. This blunted cytokine response to LPS is paralleled by the previously noted study reporting that LPS-induced TNF $\alpha$ production is decreased in cord blood monocytes and myeloid DCs of babies born to obese mothers [14]. Given the use of LPS-derivatives such as MPLA in current adjuvant development, this has implications for the obese population. With the rise in obesity in general, and particularly childhood obesity, choice of adjuvant for paediatric vaccines may gain importance to ensure the best possible efficacy is attained in the breadth of stakeholder populations. Collectively, our data highlights that neonates from obese mothers, and importantly also obese children display functional cytokine responses to the cytosolic nucleic acid Poly(I:C) demonstrating that activation of CNA sensors may have potential in future vaccine development for this at-risk population.

\section{Acknowledgements}

We would like to thank the children in Our Lady's Children's Hospital Crumlin and their parents who agreed to donate peripheral blood for this research project. We would also like to thank the women in The National Maternity Hospital at Holles St who agreed to donate cord blood.

\section{Author contributions}

KB performed experiments. BDOL, DMcL, EM \& DK identified suitable donors, collected data and bloods. KB, DC, SP, FMM, AEH \& SD designed experiments, analysed data and wrote the manuscript.

\section{Conflict of interest}

No conflict of interest.

\section{References}

[1] M.E. Falagas, M. Kompoti, Obesity and infection, Lancet Infect. Dis. 6 (7) (2006) 438-446.

[2] A. Eliakim, C. Schwindt, F. Zaldivar, P. Casali, D.M. Cooper, Reduced tetanus antibody titers in overweight children, Autoimmunity 39 (2) (2006) 137-141.

[3] D.J. Weber, W.A. Rutala, G.P. Samsa, J.E. Santimaw, S.M. Lemon, Obesity as a predictor of poor antibody response to hepatitis B plasma vaccine, JAMA 254 (22) (1985) 3187-3189.

[4] S.D. Neidich, W.D. Green, J. Rebeles, E.A. Karlsson, S. Schultz-Cherry, T.L. Noah, S. Chakladar, M.G. Hudgens, S.S. Weir, M.A. Beck, Increased risk of influenza among vaccinated adults who are obese, Int. J. Obes. (Lond.) 41 (9) (2017) 1324-1330.

[5] A. Must, S.E. Anderson, Body mass index in children and adolescents: considerations for population-based applications, Int. J. Obes. (Lond.) 30 (4) (2006) 590-594.

[6] I. Janssen, P.T. Katzmarzyk, R. Ross, Body mass index, waist circumference, and health risk: evidence in support of current National Institutes of Health guidelines, Arch. Intern. Med. 162 (18) (2002) 2074-2079.

[7] P.A. Sheridan, H.A. Paich, J. Handy, E.A. Karlsson, M.G. Hudgens, A.B. Sammon, L.A. Holland, S. Weir, T.L. Noah, M.A. Beck, Obesity is associated with impaired immune response to influenza vaccination in humans, Int. J. Obes. (Lond.) 36 (8)
(2012) 1072-1077.

[8] H.A. Paich, P.A. Sheridan, J. Handy, E.A. Karlsson, S. Schultz-Cherry, M.G. Hudgens, T.L. Noah, S.S. Weir, M.A. Beck, Overweight and obese adult humans have a defective cellular immune response to pandemic H1N1 influenza A virus, Obesity (Silver Spring) 21 (11) (2013) 2377-2386.

[9] K. Brennan, B.D. O'Leary, D. Mc Laughlin, E.P. Breen, E. Connolly, N. Ali, D.N. O'Driscoll, E. Ozaki, R. Mahony, K. Mulfaul, A.M. Ryan, A. Ni Chianain, A. McHugh, E.J. Molloy, A.E. Hogan, S. Paran, F.M. McAuliffe, S.L. Doyle, Type 1 IFN induction by cytosolic nucleic acid is intact in neonatal mononuclear cells, contrasting starkly with neonatal hyporesponsiveness to TLR ligation due to independence from endosome-mediated IRF3 activation, J. Immunol. (2018).

[10] E. Aksoy, V. Albarani, M. Nguyen, J.F. Laes, J.L. Ruelle, D. De Wit, F. Willems, M. Goldman, S. Goriely, Interferon regulatory factor 3-dependent responses to lipopolysaccharide are selectively blunted in cord blood cells, Blood 109 (7) (2007) 2887-2893.

[11] M.E. Belderbos, G.M. van Bleek, O. Levy, M.O. Blanken, M.L. Houben, L. Schuijff, J.L. Kimpen, L. Bont, Skewed pattern of Toll-like receptor 4-mediated cytokine production in human neonatal blood: low LPS-induced IL-12p70 and high IL-10 persist throughout the first month of life, Clin. Immunol. 133 (2) (2009) 228-237.

[12] D. De Wit, V. Olislagers, S. Goriely, F. Vermeulen, H. Wagner, M. Goldman, F. Willems, Blood plasmacytoid dendritic cell responses to $\mathrm{CpG}$ oligodeoxynucleotides are impaired in human newborns, Blood 103 (3) (2004) 1030-1032.

[13] D. De Wit, S. Tonon, V. Olislagers, S. Goriely, M. Boutriaux, M. Goldman, F. Willems, Impaired responses to toll-like receptor 4 and toll-like receptor 3 ligands in human cord blood, J. Autoimmun. 21 (3) (2003) 277-281.

[14] R.M. Wilson, N.E. Marshall, D.R. Jeske, J.Q. Purnell, K. Thornburg, I. Messaoudi, Maternal obesity alters immune cell frequencies and responses in umbilical cord blood samples, Pediatr. Allergy Immunol. 26 (4) (2015) 344-351.

[15] C.R. Casella, T.C. Mitchell, Putting endotoxin to work for us: monophosphoryl lipid A as a safe and effective vaccine adjuvant, Cell. Mol. Life Sci. 65 (20) (2008) 3231-3240.

[16] G.T. Jennings, M.F. Bachmann, Immunodrugs: therapeutic VLP-based vaccines for chronic diseases, Annu. Rev. Pharmacol. Toxicol. 49 (2009) 303-326.

[17] J.D. Marshall, D. Higgins, C. Abbate, P. Yee, G. Teshima, G. Ott, T. dela Cruz, D. Passmore, K.L. Fearon, S. Tuck, G. Van Nest, Polymyxin B enhances ISS-mediated immune responses across multiple species, Cell Immunol. 229 (2) (2004) 93-105.

[18] C. Tagliabue, N. Principi, C. Giavoli, S. Esposito, Obesity: impact of infections and response to vaccines, Eur. J. Clin. Microbiol. Infect Dis. 35 (3) (2016) 325-331.

[19] T.R. Kollmann, J. Crabtree, A. Rein-Weston, D. Blimkie, F. Thommai, X.Y. Wang, P.M. Lavoie, J. Furlong, E.S. Fortuno 3rd, A.M. Hajjar, N.R. Hawkins, S.G. Self, C.B. Wilson, Neonatal innate TLR-mediated responses are distinct from those of adults, J. Immunol. 183 (11) (2009) 7150-7160.

[20] T.R. Kollmann, O. Levy, R.R. Montgomery, S. Goriely, Innate immune function by Toll-like receptors: distinct responses in newborns and the elderly, Immunity 37 (5) (2012) 771-783.

[21] E. Carolan, A.E. Hogan, M. Corrigan, G. Gaotswe, J. O'Connell, N. Foley, L.A. O'Neill, D. Cody, D. O'Shea, The impact of childhood obesity on inflammation, innate immune cell frequency, and metabolic microRNA expression, J. Clin. Endocrinol. Metab. 99 (3) (2014) E474-E478.

[22] C.L. Ogden, M.D. Carroll, C.D. Fryar, K.M. Flegal, Prevalence of obesity among adults and youth: United States, 2011-2014, NCHS Data Brief (219) (2015) 1-8.

[23] K.M. Flegal, D. Kruszon-Moran, M.D. Carroll, C.D. Fryar, C.L. Ogden, Trends in obesity among adults in the United States, 2005 to 2014, JAMA 315 (21) (2016) 2284-2291.

[24] S.L. Prescott, A. Taylor, J. Roper, A. Wahdan, P. Noakes, C. Thornton, J. Dunstan, J.W. Upham, Maternal reactivity to fetal alloantigens is related to newborn immune responses and subsequent allergic disease, Clin. Exp Allergy 35 (4) (2005) 417-425.

[25] D.B. Tse, B.K. Young, Co-ordinate expression of Th1/Th2 phenotypes in maternal and fetal blood: evidence for a transplacental nexus, J. Perinat. Med. 40 (2) (2012) 165-170.

[26] M. Halonen, I.C. Lohman, D.A. Stern, A. Spangenberg, D. Anderson, S. Mobley, K. Ciano, M. Peck, A.L. Wright, Th1/Th2 patterns and balance in cytokine production in the parents and infants of a large birth cohort, J. Immunol. 182 (5) (2009) 3285-3293.

[27] A.K. Larsson, C. Nilsson, A. Hoglind, E. Sverremark-Ekstrom, G. Lilja, M. TroyeBlomberg, Relationship between maternal and child cytokine responses to allergen and phytohaemagglutinin 2 years after delivery, Clin. Exp. Immunol. 144 (3) (2006) 401-408.

[28] M. Lappalainen, M. Roponen, J. Pekkanen, K. Huttunen, M.R. Hirvonen, Maturation of cytokine-producing capacity from birth to $1 \mathrm{yr}$ of age, Pediatr. Allergy Immunol. 20 (8) (2009) 714-725.

[29] J. Crouse, U. Kalinke, A. Oxenius, Regulation of antiviral T cell responses by type I interferons, Nat. Rev. Immunol. 15 (4) (2015) 231-242.

[30] P. Rizza, F. Moretti, I. Capone, F. Belardelli, Role of type I interferon in inducing a protective immune response: perspectives for clinical applications, Cytokine Growth Factor Rev. 26 (2) (2015) 195-201.

[31] Y. Wu, B. Yue, J. Liu, Lipopolysaccharide-induced cytokine expression pattern in peripheral blood mononuclear cells in childhood obesity, Mol. Med. Rep. 14 (6) (2016) 5281-5287. 